\title{
First stages of plasticity in three-point bent Au nanowires detected by in situ Laue microdiffraction
}

Cite as: Appl. Phys. Lett. 116, 243101 (2020); https://doi.org/10.1063/5.0012816

Submitted: 05 May 2020 . Accepted: 28 May 2020 . Published Online: 15 June 2020

Z. Ren, (D) T. W. Cornelius, C. Leclere, A. Davydok, (D) J.-S. Micha, (i) O. Robach, G. Richter, and (D) O. Thomas
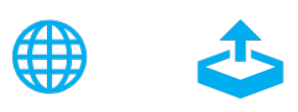

\section{ARTICLES YOU MAY BE INTERESTED IN}

Magnetic-field imaging using vortex-core MFM tip

Applied Physics Letters 116, 242406 (2020); https://doi.org/10.1063/5.0010623

An elementary photo-thermoelectric transistor: Experimental demonstration

Applied Physics Letters 116, 243501 (2020); https://doi.org/10.1063/5.0010264

Intrinsic role of $\uparrow \uparrow \downarrow \downarrow$-type magnetic structure on magnetoelectric coupling in $\mathrm{Y}_{2} \mathrm{NiMnO}_{6}$

Applied Physics Letters 116, 242901 (2020); https://doi.org/10.1063/5.0009568

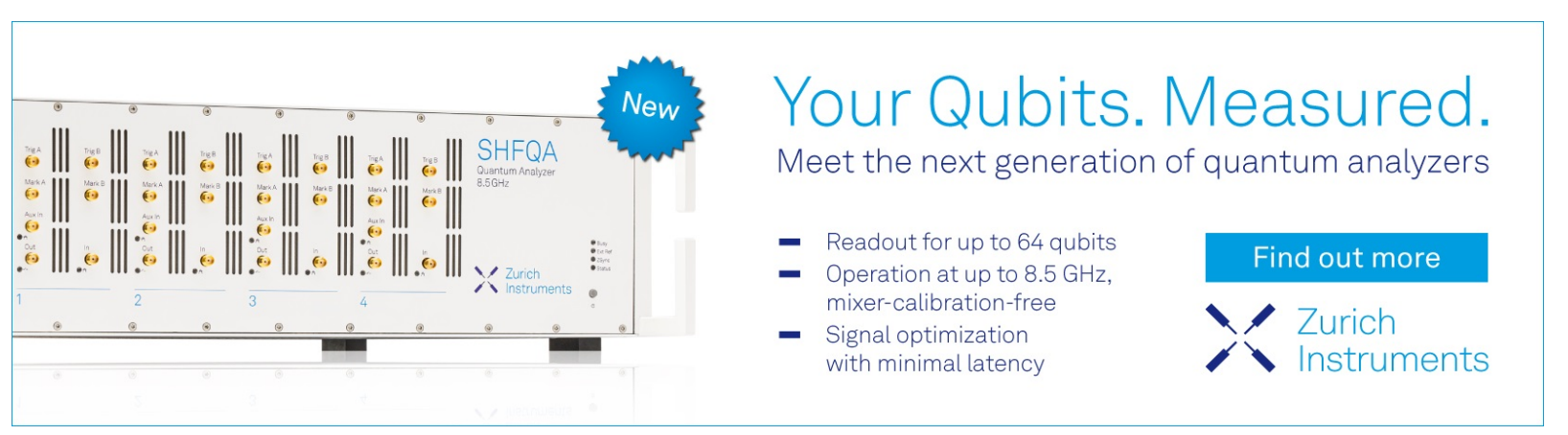




\title{
First stages of plasticity in three-point bent $\mathrm{Au}$ nanowires detected by in situ Laue microdiffraction
}

Cite as: Appl. Phys. Lett. 116, 243101 (2020); doi: 10.1063/5.0012816

Submitted: 5 May 2020 - Accepted: 28 May 2020 •

Published Online: 15 June 2020

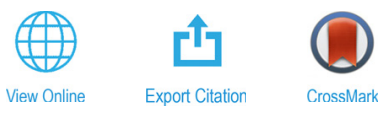

Z. Ren, ${ }^{1,2}$ T. W. Cornelius, ${ }^{1, a)}$ (D) C. Leclere, ${ }^{1}$ A. Davydok, ${ }^{1,3}$ J.-S. Micha,, ${ }^{4,5}$ (D) O. Robach, ${ }^{4,5}$ (iD G. Richter, and O. Thomas ${ }^{\top}$ iD

\author{
AFFILIATIONS \\ ${ }^{7}$ Aix Marseille Univ, Univ Toulon, CNRS, IM2NP, 13397 Marseille, France \\ ${ }^{2}$ Deutsches Elektronen-Synchrotron (DESY), Notkestrasse 85, 22607 Hamburg, Germany \\ ${ }^{3}$ Helmholtz-Zentrum Geesthacht, Notkestrasse 85, 22607 Hamburg, Germany \\ ${ }^{4}$ CRG-IF BM32 Beamline at the European Synchrotron (ESRF), CS4O220, 38043 Grenoble Cedex 9, France \\ ${ }^{5}$ University Grenoble Alps, CNRS, CEA-IRIG, 38054 Grenoble, France \\ ${ }^{6}$ Max Planck Institute for Intelligent Systems, Heisenbergstrasse 3, 70569 Stuttgart, Germany \\ a) Author to whom correspondence should be addressed: thomas.cornelius@im2np.fr
}

\begin{abstract}
The first stages of plasticity in three-point bent Au nanowires are investigated by in situ three-point bending tests in combination with Laue micro-diffraction. To separate the elastic and plastic deformation, loading-unloading cycles were performed with increasing load in each consecutive cycle. The storage of the first four geometrically necessary dislocations of $[01 \overline{1}](111)$ slip system is observed in the vicinity of both clamping points, which might be attributed to the local rotations induced by the rigid Si support. At later stages of the deformation, additional slip systems are activated either by the torsion of the nanowire or by unintentional indentation from the AFM tip. The cyclic loading-unloading approach combined with Laue microdiffraction thus allows to study the onset of plasticity in defect-scarce nanostructures deformed by bending, offering additional possibilities in studying the dislocation nucleation process in bent nano-objects, which are essential for future applications, e.g., in flexible electronics and nano-electromechanical systems.
\end{abstract}

Published under license by AIP Publishing. https://doi.org/10.1063/5.0012816

In 2004, Uchic et al. pioneered the field of micro-compression testing on focused ion beam (FIB) fabricated metal micropillars, revealing a trend that became known as "smaller is stronger." ${ }^{\text {. Since }}$ then, the number of studies on the mechanical properties of microand nanostructures increased tremendously. ${ }^{2,3}$ While the first experiments focused on the ex situ characterization of the plasticity at the microscale, ${ }^{1}$ recent works concentrate more on the in situ monitoring of the deformation by scanning electron microscopy, ${ }^{4}$ transmission electron microscopy, ${ }^{5-7}$ or synchrotron X-ray diffraction techniques, including Laue micro-diffraction ${ }^{9-11}$ and Bragg coherent X-ray diffraction imaging. 12,13

The very large elastic energy that may be stored in nanostructures strained up to the theoretical limit of elasticity of the material may favor the nucleation of dislocations. The nucleation of the first dislocations in defect-scarce nanostructures has recently attracted considerable attention. ${ }^{14-16}$ In the case of tensile tested single-crystalline defect-scarce $\mathrm{Au}$ nanowires, partial dislocations with the highest Schmid factors were demonstrated to be nucleated from the free surface and rapidly glide through the nanowire leading to twin mediated deformation. ${ }^{6,7}$ In contrast to the numerous studies on tensile tested nanowires in existing literature, ${ }^{5,14,15}$ bending-induced plastic deformation is not well investigated despite its importance for applications such as flexible electronics or micro-electromechanical systems. Most of the works on bending experiments concentrated on FIB-milled cantilever beams. ${ }^{17,18}$ The influence of strain gradients on the dislocation nucleation, however, remains unclear. In 2012, Schopf et al. observed ultrahigh yield strength during the cantilever bending of copper nanowires, which they attributed to the nucleation of partial dislocations. ${ }^{19}$ For Au nanowires bent as a consequence of buckling ${ }^{6}$ and for $\mathrm{Ni}$ nanowires bent by a colloidal thin film under electron beam irradiation, ${ }^{20}$ the storage of dislocations and the formation of low angle grain boundaries were reported, respectively. Some recent MD simulations 
on the bending of $\mathrm{Au}$ nanowires, ${ }^{21}$ on the other hand, suggest that, similar to uniaxial tensile tests, partial dislocations nucleate from the surface leading to the formation of wedge-shaped twins, which accommodate the nanowire bending and completely vanish upon unloading.

In a previous work, ${ }^{11}$ we reported on the mechanical behavior of $\mathrm{Au}$ nanowires loaded in a three-point bending configuration monitored in situ by Laue micro-diffraction, revealing the activation of unexpected slip systems and the storage of geometrically necessary dislocations (GNDs), which is attributed to the dislocation nucleation process at the clamping points. While these studies concentrated on severely plastically deformed nanowires, the present work focuses on the first stages of plasticity in three-point bent nominally defect-free Au nanowires that were cyclically loaded and unloaded to separate the influence from the elastic strain and defects. In a seemingly elastic regime, the storage of the first GNDs is revealed by the splitting of the Laue diffraction peaks at both clamping points.

The in situ three-point bending experiments are performed on the BM32 beamline at the European Synchrotron ESRF in Grenoble (France). For this purpose, the in situ AFM "SFINX" was installed on the sample stage, ${ }^{22}$ which is inclined by $40^{\circ}$ with respect to the incident polychromatic X-ray beam. The polychromatic X-ray beam with an energy bandwidth ranging from 5 to $25 \mathrm{keV}$ was focused down to $500 \mathrm{~nm}(\mathrm{~V}) \times 500 \mathrm{~nm}(\mathrm{H})$ on the sample surface using a pair of Kirkpatrick-Baez $(\mathrm{KB})$ mirrors. The $\mathrm{Au} \mathrm{L}_{\mathrm{III}}$ fluorescence yield was monitored using a Röntec XFlash energy-dispersive detector, and the Laue micro-diffraction patterns were recorded by a MarCCD detector with a pixel size of $80 \mu \mathrm{m}$, which was installed at an angle of $90^{\circ}$ and at a distance of $70 \mathrm{~mm}$ from the sample position.

Single-crystalline nominally defect-free gold nanowires were grown by vapor phase deposition on carbon-coated tungsten substrates. ${ }^{14}$ The as-grown nanowires are deposited on a Si surface patterned with $10 \mu \mathrm{m}$ wide and $1.5 \mu \mathrm{m}$ deep micro-trenches, employing the same procedures as described elsewhere. ${ }^{10,11}$ As illustrated by the SEM image in Figs. 1(a) and 1(b), the Au nanowire used in this experiment (length: $23.7 \mu \mathrm{m}$, width: $0.7 \mu \mathrm{m}$ ) crosses two micro-trenches and lies almost perpendicular to the Si supports. According to the previous studies, ${ }^{10}$ the nanowire growth direction is $[01 \overline{1}]$ and the loading direction is [111]. Prior to the nano-mechanical test, the SFINX-tip and the focused X-ray beam were positioned in the center of the left trench using AFM topography and scanning X-ray fluorescence mapping, respectively. Lowering the SFINX tip with a constant speed of $1.5 \mathrm{~nm} / \mathrm{s}$, the tip pushes against the suspended nanowire and deflects it. To separate the elastic and plastic deformation, three loading-unloading cycles were performed with increasing load in each consecutive cycle, as schematically illustrated in Fig. 1(c). At each loading and after each unloading, a 2D X-ray diffraction map of the suspended nanowire was recorded, employing the $\mathrm{KB}$ scan approach. ${ }^{23}$ Here, the focused X-ray beam is scanned by translating the horizontal (along the nanowire direction) and the vertical (perpendicular to the nanowire direction) $\mathrm{KB}$ mirror in steps of $1 \mu \mathrm{m}$ and $0.2 \mu \mathrm{m}$, respectively, thus measuring the complete deformation profile of the nanowire without introducing any vibrations to the nano-mechanical setup.

The diffraction patterns related to the Au nanowire are extracted from the $2 \mathrm{D} \mathrm{KB}$-maps using the positions with the maximal $\mathrm{Au} \mathrm{L}_{\mathrm{III}}$ fluorescence yield. Subtracting background diffraction patterns
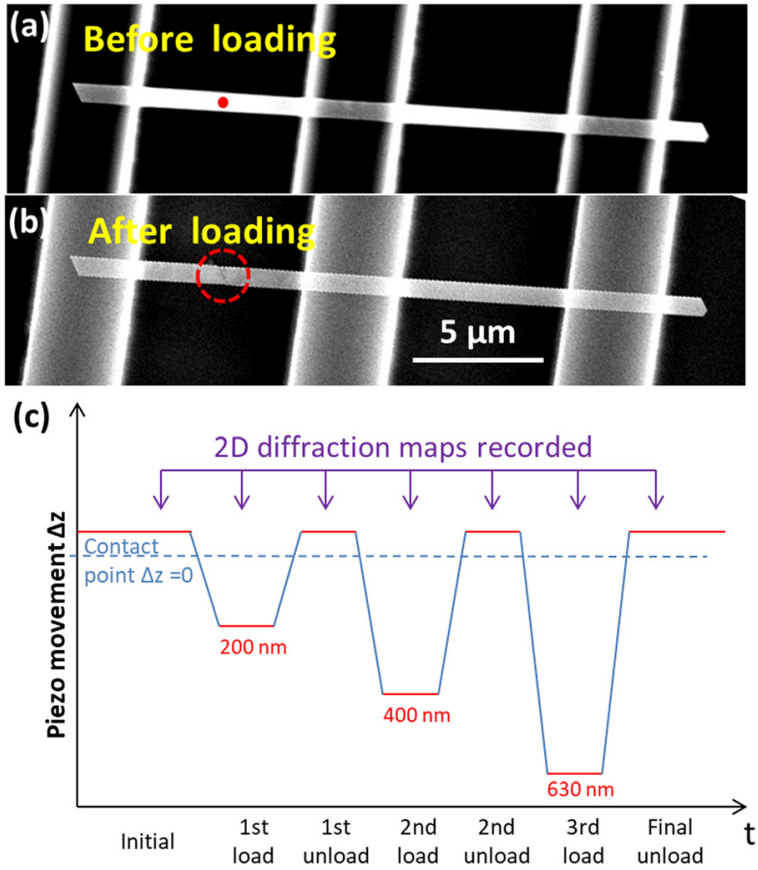

FIG. 1. SEM image of the Au nanowire (a) before and (b) after plastic deformation. (c) Schematic of the loading-unloading sequence. The red lines represent the stages where 2D Laue micro-diffraction maps are recorded.

recorded at the nearby bare $\mathrm{Si}$ substrate, the $\mathrm{Si}$ diffraction peaks are removed, increasing the visibility of the Au nanowire Laue spots, which were indexed by the LaueTools software. ${ }^{24}$ The background corrected diffraction patterns at one loading stage are then summed up, generating an integrated diffraction pattern, which represents a complete orientation profile for one deformation state. ${ }^{11}$

The integrated diffraction pattern of the initial state is presented in Fig. 2(a). The well-defined integrated diffraction peaks with a minor spread signify that the Laue spots essentially remain at the same positions on the detector for all the points measured along the nanowire, i.e., the nanowire is perfectly straight. The small spread is due to defects induced by a test loading performed prior to the experiment. The integrated Laue pattern is composed of a set of parallelograms located at each original Laue spot on the detector, as illustrated in Fig. 2(b). Concentrating on the Au 331 diffraction peak displayed in the inset of Fig. 2(b), the horizontal and vertical displacements (highlighted by the red and black lines, respectively) originate from bending and torsion of the nanowire, which induce a rotation of the crystal around the $\mathrm{Au}[211]$ and the $\mathrm{Au}[01 \overline{1}]$ axes, respectively. ${ }^{1}$

The evolution of the integrated diffraction peak is exemplified on the Au 331 Laue spot in Fig. 2(c), illustrating the deformation process during cyclic loading-unloading (more information can be found in the supplementary material). During three-point bending, the integrated Laue spots describe parallelograms, whose width and height increase with increasing load, i.e., from one loading-unloading cycle to the next. From the dimensions of the parallelogram's shape, the bending angle is inferred to be $5.2,9.6$, and $11.6^{\circ}$ for the three consecutive loads, while the maximum torsion increases from 6.4 to 7.5 and then to $9.5^{\circ}$. After the first and the second loading-unloading cycle, 

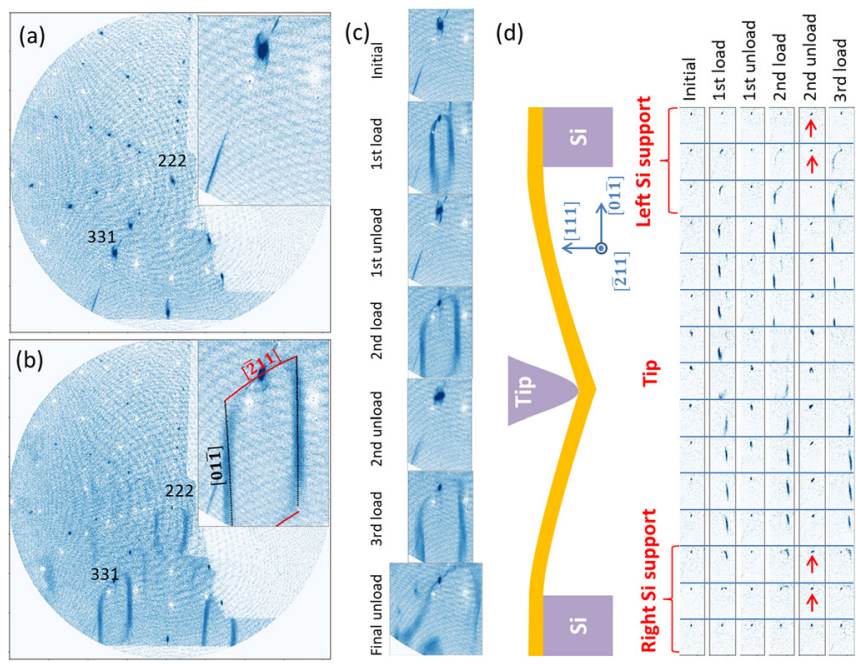

FIG. 2. Integrated Laue diffraction pattern of the Au nanowire (a) in its initial state and (b) during three-point bending test. Inset: an enlarged area around the Au 331 Laue spot. The expected movements of the Laue spot on the detector for pure bending (rotation around $\mathrm{Au}[\overline{211}]$ ) and for pure torsion (rotation around $\mathrm{Au}$ [011]) are indicated as red and black lines, respectively. (c) Integrated Au 331 Laue diffraction peak during the cyclic loading-unloading process. (d) Schematic of the three-point bending of the Au nanowire and position-dependent representation of the $\mathrm{Au} 331$ Laue spot recorded by KB-scans during the cyclic loading-unloading. The red arrows highlight the appearance of split Laue diffraction peaks.

the integrated diffraction peaks return to roughly a single spot indicating that the nanowire returned to its initial state and that the deformation was essentially elastic. After the third loading-unloading cycle, however, the integrated Laue diffraction pattern exhibits strongly blurred diffraction peaks that evidence severe plastic deformation of the nanowire (see Fig. S1 in the supplementary material).

While the nanowire remains essentially straight after the first two cycles, the onset of plasticity can already be observed at both clamping points, as well as around the loading position. To illustrate this, the $\mathrm{Au}$ 331 Laue spot is displayed separately for each measurement position along the nanowire in Fig. 2(d). Near the loading position, the Laue diffraction peaks are strongly blurred during loading, which corresponds to the bending-induced strain gradient in the nanowire. After unloading, the "comma shaped" diffraction peak remains slightly spread, which is probably caused by the unintentional indentation of the nanowire with the SFINX-tip. More and more diffraction peaks are extended with the increasing number of loading-unloading cycles, indicating a spread of these local defects along the nanowire. In between the Si supports and the loading point, the diffraction peaks are essentially elongated vertically during three-point bending, which corresponds to the nanowire torsion. However, after unloading, the diffraction peaks recover their initial sharpness, demonstrating that this part of the nanowire remains basically defect-free. In the vicinity of the Si supports, the diffraction peaks mainly show a horizontal movement during loading, i.e., that the nanowire is deformed predominantly by bending. After the first loading-unloading cycle, the Au 331 Laue spots are at the same position on the detector and have the same shape as before, whereas split Laue spots are apparent at both clamping points after the second cycle, as indicated by the red arrows in Fig. 2(d). As pointed out by Hofmann et al., ${ }^{25}$ while for bulk materials, split Laue spots typically indicate the presence of low angle grain boundaries, a small number of stored GNDs is sufficient to form similar structures in nano-objects.

The splitting of the Laue spots and the related storage of GNDs were further evaluated by the indexation of the Laue diffraction patterns [as the one shown in Fig. 3(a)] recorded in the vicinity of the clamping positions. As illustrated by the decomposed rotation [see Figs. 3(b) and 3(c)] of the nanowire crystal around the [111] top surface direction, the $[01 \overline{1}]$ nanowire growth axis, and the $[\overline{2} 11]$ direction perpendicular to the loading plane, the crystal rotates around the $[\overline{2} 11]$ axis by about $1^{\circ}$. This rotation coincides with the local rotations induced during the elastic bending. Considering the edge dislocations for the 12 slip systems existing in FCC crystals, a rotation around the $[\overline{2} 11]$ axis corresponds to the storage of $[01 \overline{1}](111)$ type edge dislocations in the minimum energy configuration. The minor deviation of the data points from the calculation is probably caused by uncertainties in defining the exact locations of the Laue spots on the detector.

According to $\mathrm{Nye}^{26}$ the rotation angle $\Delta \theta$ is directly related to the dislocation density $\rho_{\mathrm{GND}}$ in the probed volume by

$$
\Delta \theta=\rho_{G N D} \cdot L \cdot b .
$$

Here, $b$ is Burger's vector and $L$ is the length of the probed volume along $b$. The absolute number of dislocations within the probed volume is estimated from $\rho_{\mathrm{GND}}$ by the following equation:

$$
\mathrm{n}=\rho_{G N D} \cdot L \cdot t
$$

where $t$ is the nanowire thickness. Combining Eqs. (1) and (2) yields

$$
n=\Delta \theta \cdot t / b .
$$

Considering a nanowire thickness of $60 \mathrm{~nm}$ (see the FEM simulation in the supplementary material), the lattice parameter of gold of $0.408 \mathrm{~nm}$, and the measured crystal rotation of $1^{\circ}$ results in about four GNDs stored in the probed volume.

Previous three-point bending experiments focusing on severely plastically deformed $\mathrm{Au}$ nanowires ${ }^{11}$ evidenced the activation of $[01 \overline{1}](111)$ and $[101](\overline{1} 11)$ slip systems and the storage of the corresponding GNDs. The present work further explores the very first
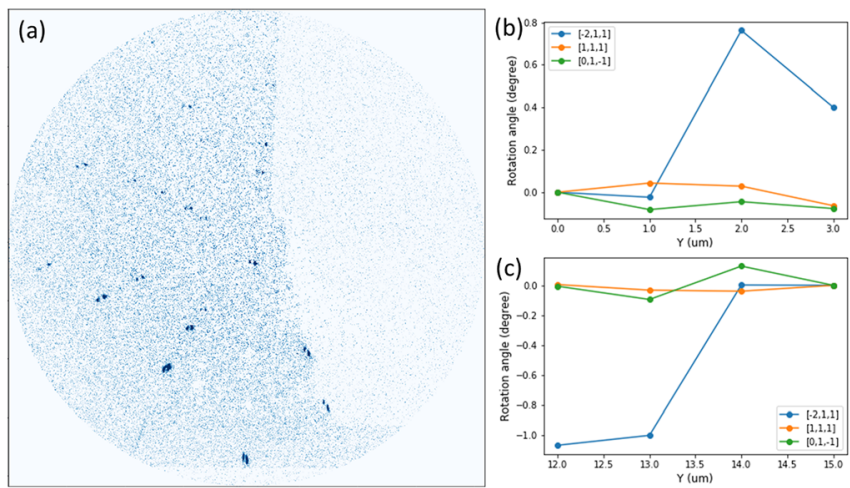

FIG. 3. (a) Laue diffraction pattern recorded in the vicinity of the left clamping point after the second loading-unloading cycle showing split Laue spots. Onedimensional orientation maps (c) at the left and (d) the right Si supports. 
stages of plasticity in three-point bent nanowires. The first signs of defects are observed both at the loading position and in the vicinity of the clamping points in the seemingly elastic regime. At these three positions, the highest stress concentrations are located, as demonstrated by finite element method (FEM) simulations (see the supplementary material). While the applied force is not directly accessible experimentally, the FEM simulations yield an applied force of 200 and $680 \mathrm{nN}$ for the first and second loadings, respectively. The maximum von-Mises stress in the second loading cycle, where the first GNDs were found to be nucleated and stored in the vicinity of the clamping points, thus corresponding to the yield strength of the nanowire, amounts to $0.48 \mathrm{GPa}$. This value is in good agreement with tensile tests on similar nanowire reported in the literature, yielding yield strengths ranging from 0.4 to $1.6 \mathrm{GPa}^{27}$

The nanowire was found to be predominantly deformed by bending in the vicinity of the clamping points, where the elastic deformation and the activated $[01 \overline{1}](111)$ slip system induce the same local rotation. Note that the orientation of the nanowire with respect to the Si ridges may have an impact on the activation of specific slip systems by imposing a certain shape on the nanowire and thus local crystal rotations due to their higher rigidity compared with that of gold. Extensive torsion was evidenced in other sections of the nanowire, as well as the unintentional indentation at the loading point, which may eventually result in the activation of further slip systems at later deformation stages. In the present work, however, the additionally activated slip systems could not be determined due to the very weak diffraction intensity of the plastically deformed nanowire after the third loading-unloading cycle (as illustrated by Fig. S1 in the supplementary material). Further experiments are needed to probe the dislocation multiplication process in defect-scarce nanostructures during threepoint bending tests.

In conclusion, the very first stages of plasticity in three-point bent gold nanowires were monitored by in situ Laue micro-diffraction. Successive loading and unloading of the nanowire allowed for deconvoluting of the elastic strain induced broadening of the Laue spots and the GNDs caused streaking. In a seemingly elastic regime, the nucleation and storage of dislocations are observed at around the loading position and in the vicinity of the clamping points, where the stress is the highest. The storage of the first four GNDs of the $[01 \overline{1}](111)$ type was evidenced by the splitting of the Laue diffraction spots by about $1^{\circ}$ in the vicinity of the clamping points. The rotation induced by the GNDs coincides with the local deformation during elastic bending. The maximum von Mises stress corresponding to the yield strength of the Au nanowire amounts to $0.48 \mathrm{GPa}$, which is in good agreement with the literature values on uniaxial tensile tests on $\mathrm{Au}$ nanowires. ${ }^{27}$ Additional slip systems are activated at later stages of the deformation induced either by the torsion of the nanowire or by unintentional indentation from the AFM tip. As demonstrated, the cyclic loadingunloading approach combined with Laue microdiffraction paves the way to study the onset of plasticity in defect-scarce nanostructures deformed by bending and allows for resolving the activated slip systems, as well as for determining the absolute number of stored GNDs. Hence it offers additional possibilities in studying the dislocation nucleation process in bent nano-objects, providing further insights into the understanding of plasticity at the nanoscale, which are essential for future applications, e.g., in flexible electronics and nanoelectromechanical systems.
See the supplementary material for further information about the Laue micro-diffraction pattern of the deformed nanowire after the third unloading, and the shape of the diffraction peaks after each unloading and finite element method (FEM) simulations of the threepoint bending of the nanowire.

The authors gratefully acknowledge the financial support from the French National Research Agency through Project No. ANR11-BS10-0014 MecaniX. They further thank the French CRG program committee for the allocated beamtime at the BM32 beamline at ESRF and L. Belliard at INSP in Paris (France) for providing the Si micro-trenches.

\section{DATA AVAILABILITY}

The data that support the findings of this study are available within the article and its supplementary material.

\section{REFERENCES}

${ }^{1}$ M. D. Uchic, D. M. Dimiduk, J. N. Florando, and W. D. Nix, Science 305, 986-989 (2004).

${ }^{2}$ S. Wang, Z. Shan, and H. Huang, Adv. Sci. 4, 1600332 (2017).

${ }^{3}$ M. D. Uchic, P. A. Shade, and D. M. Dimiduk, Annu. Rev. Mater. Res. 39, 361-386 (2009).

${ }^{4}$ D. Kiener, W. Grosinger, G. Dehm, and R. Pippan, Acta Mater. 56, 580-592 (2008)

${ }^{5}$ S. Lee, J. Im, Y. Yoo, E. Bitzek, D. Kiener, G. Richter, B. Kim, and S. H. Oh, Nat. Commun. 5, 3033 (2014).

${ }^{6}$ B. Roos, B. Kapelle, G. Richter, and C. A. Volkert, Appl. Phys. Lett. 105, 201908 (2014).

${ }^{7}$ J. Wang, Z. Zeng, C. R. Weinberger, Z. Zhang, T. Zhu, and S. X. Mao, Nat. Mater. 14, 594-600 (2015).

${ }^{8}$ T. W. Cornelius and O. Thomas, Prog. Mater. Sci. 94, 384-434 (2018).

${ }^{9}$ C. Kirchlechner, P. J. Imrich, W. Grosinger, M. W. Kapp, J. Keckes, J. S. Micha, O. Ulrich, O. Thomas, S. Labat, C. Motz, and G. Dehm, Acta Mater. 60, 1252-1258 (2012).

${ }^{10}$ C. Leclere, T. W. Cornelius, Z. Ren, A. Davydok, J.-S. Micha, O. Robach, G. Richter, L. Belliard, and O. Thomas, J. Appl. Crystallogr. 48, 291-296 (2015).

${ }^{11}$ Z. Ren, T. W. Cornelius, C. Leclere, A. Davydok, J.-S. Micha, O. Robach, G. Richter, and O. Thomas, J. Appl. Phys. 124, 185104 (2018).

${ }^{12}$ A. Davydok, T. Cornelius, Z. Ren, C. Leclere, G. Chahine, T. Schülli, F. Lauraux, G. Richter, and O. Thomas, Quantum Beam Sci. 2, 24 (2018).

${ }^{13}$ M. Dupraz, G. Beutier, T. W. Cornelius, G. Parry, Z. Ren, S. Labat, M.-I. Richard, G. A. Chahine, O. Kovalenko, M. De Boissieu, E. Rabkin, M. Verdier, and O. Thomas, Nano Lett. 17, 6696-6701 (2017).

${ }^{14}$ G. Richter, K. Hillerich, D. S. Gianola, R. Mönig, O. Kraft, and C. A. Volkert, Nano Lett. 9, 3048-3052 (2009).

${ }^{15}$ L. Y. Chen, G. Richter, J. P. Sullivan, and D. S. Gianola, Phys. Rev. Lett. 109, 125503 (2012).

${ }^{16}$ Z. Sun, C. Huang, J. Guo, J. T. Dong, R. F. Klie, L. J. Lauhon, and D. N. Seidman, ACS Nano 13, 3730-3738 (2019).

${ }^{17}$ W. Luo, C. Kirchlechner, X. Fang, S. Brinckmann, G. Dehm, and F. Stein, Mater. Des. 145, 116-121 (2018).

${ }^{18}$ C. Kirchlechner, P. J. Imrich, W. Liegl, J. Pörnbacher, J. S. Micha, O. Ulrich, and C. Motz, Acta Mater. 94, 69-77 (2015).

${ }^{19}$ C. Schopf, M. Schamel, H. P. Strunk, and G. Richter, Adv. Eng. Mater. 14, 975-980 (2012)

${ }^{20}$ L. Wang, D. Kong, Y. Zhang, L. Xiao, Y. Lu, Z. Chen, Z. Zhang, J. Zou, T. Zhu, and X. Han, ACS Nano 11, 12500-12508 (2017).

${ }^{21}$ W. G. Nöhring, J. J. Möller, Z. Xie, and E. Bitzek, Extreme Mech. Lett. 8, 140-150 (2016).

${ }^{22}$ Z. Ren, F. Mastropietro, A. Davydok, S. Langlais, M.-I. Richard, J.-J. Furter, O. Thomas, M. Dupraz, M. Verdier, G. Beutier, P. Boesecke, and T. W. Cornelius, J. Synchrotron Radiat. 21, 1128-1133 (2014) 
${ }^{23}$ C. Leclere, T. W. Cornelius, Z. Ren, O. Robach, J.-S. Micha, A. Davydok, O. Ulrich, G. Richter, and O. Thomas, J. Synchrotron Radiat. 23, 1395-1400 (2016).

${ }^{24}$ J.-S. Micha, "Lauetools: Open source Python packages for x-ray microlaue diffraction analysis," http://sourceforge.net/projects/lauetools (2014).
${ }^{25}$ F. Hofmann, B. Abbey, W. Liu, R. Xu, B. F. Usher, E. Balaur, and Y. Liu, Nat. Commun. 4, 2774 (2013).

${ }^{26}$ J. Nye, Acta Metall. 1, 153-162 (1953).

${ }^{27}$ A. Sedlmayr, E. Bitzek, D. S. Gianola, G. Richter, R. Mönig, and O. Kraft, Acta Mater. 60, 3985-3993 (2012). 